\title{
Tackling the Complexity of Future Networks
}

\author{
Takashi Egawa, Yoshiaki Kiriha, and Akira Arutaki \\ System Platforms Research Laboratories, NEC Corporation, \\ Shimonumabe 1753, Nakahara-ku, Kawasaki, Kanagawa, 211-8666 Japan \\ \{t-egawa@ct,y-kiriha@ay, aarutaki@bp\}.jp.nec.com
}

\begin{abstract}
Though the Internet succeeded to converge data and voice networks, it itself is diverging now. The authors believe this derives from two facts; one is the end of universal service era thanks to abundant communication services, and the other the fusion of computers and communications because the speed of networks is catching up that of computer's internal bus. These facts allow us to build networks proprietary for specific purposes such as sensor networks or web services for e-commerce where computer and communication technologies are tightly integrated. As a result, networks are becoming enormously complicated and heterogeneous, and without our effort they will become uncontrollable. The authors believe active network technologies were a good try, but it was not enough. Theories build on solid mathematical basis is indispensable for the analysis of huge systems, and we should try to build such theory. Complex systems theory provides various mathematical formulas such as 'scale-free', and it can be a good starting point. We show as an example that by using selforganization theory ubiquitous networks are considered indispensable for system stability in future business network environment.
\end{abstract}

\section{Introduction}

The network is diverging again.

Convergence of various networks, data and voice networks in particular was a dream of network engineers for many years. Huge amount of efforts such as ISDN or ATM were made, and finally IP succeeded to grasp the Holy Grail. It succeeded to integrate voice and data traffic. Layer three function, a function to identify a node in network-wide manner and to deliver data to the node, has been unified with IP in most important wired networks, and wireless networks will join when 4G mobile communication service starts.

However, dividing and diverging of IP network itself are under way. As shown in the end-to-end argument [1], IP networks was originally designed so that IP layer functions would deliver packets from end to end, and each end host controls data delivery by doing flow controls, retransmissions, or encryptions/decryptions. This is not the case any more. Network Address Translators (NATs) and virtual IP/virtual server technologies used in load balancers have destroyed the uniform address space. Firewalls have introduced application layer controls into IP packet flows, which make it possible to interfere policies or intentions of organizations in data delivery. IETF OPES WG enumerates various boxes possible to insert as an intermediary in the 
end-to-end loop [2]. One of them, TCP performance enhancement box [3], cuts off the feedback loop of TCP flow control and tries to increase the performance without the modification of end hosts. This is convenient for novice users, but this also means that the performance of TCP/IP communications with my PC does not increase even if I update the protocol stack of my PC.

Divergence of overlay networks on IP networks is even larger. There are many application specific networks such as P2Ps, x-bones, intranets, extranets, and the web. They have their proprietary address space and routing mechanisms. Some of them are unicast, and some of them are multicast. Even the property of connections may differ: connections between web sites, i.e., links, are unidirectional. Convergence of these various networks seems impossible in the near future. And active network technologies have been trying to make divergence easier.

Why such things happened? We believe this derives from the abundance of excellent networks. The abundance ended our desire for universal service, and we are now on a new stage where differentiation is more important. The excellence of communication service is accelerating the fusion of computer world and communication world, especially through the fact that the bandwidth of Ethernet is catching up with that of computers' internal bus.

The problem is, without our enormous effort this divergence would lead us to chaotic world where security breaches are common, root causes of failures or QoS degradation are unresolved, and routing tables never converge.

This paper is organized as follows. We discuss the cause of the divergence, abundance, in details in chapter 2 , and excellence in chapter 3 . Then in chapter 4 we propose to introduce knowledge from other areas to solve these issues. The theory of scale-free from complexity system theory is introduced, and why ubiquitous network is indispensable in future business network environment is explained using selforganization theory. Chapter 5 concludes this paper.

\section{Abundance of Communication Services}

People believed for many years that telecommunication services were different from other goods. Telecommunication carriers were considered as natural monopolies, and in compensation of monopoly status they had the obligation to provide, and they proudly provided, communication services universally [12]. The phrase 'anytime, anywhere, with anybody' well speaks the ideal.

However, as communication technologies evolve, it became easier to provide communication services. The precious service became common, and then abundant. The carrier's special status gradually diminished. Telecommunication carriers were privatized, and market mechanism was introduced in many countries. The initial cost to enter into the market decreased drastically. Startups provide public Wi-Fi infrastructure in some cities today. A person who installs LAN in his house is not very rare.

The necessity and the desire for universal service also diminished. Basic POTS service is available at anywhere in many countries. Best-effort IP service can easily be obtained, and it is not the target of universal service policy. 
Abundance brings us to a new stage of economy where important properties of the industry differ, as Rostow claimed in [4]. The driving force of telecommunication services, and network technologies as well, changed from public sectors to private sectors. This changed the core competence from standardization and universality to differentiation and customization.

In business sector communication services are tools to do business. They must be optimized for business processes, and as long as the business requirements are satisfied the service must be cheap as much as possible. This leads us to various customizations. How a call should be transferred to the right personnel (is it OK for an executive to answer a phone call to another executive if all secretaries are out of office)? Reliability is always preferable, but the acceptable cost differs. Authentication for multicast clients is indispensable for applications of some companies, but is not for others.

In consumer sector various customization also exists. 'What sort of phone you carry and how you customize it says a great deal about you, just as the choice of car did for a previous generation', wrote the Economist [5]. The market size of customizing ring tones was Y85b (\$770m) in Japan in 2002, which occupies $0.8 \%$ of the all IT sectors, and it will become Y150b (\$1.4b) in 2007 [6]. In early days of short mails, the sales of a mobile phone was three times larger than others, because it could use heart mark [7]. The features of cameras (how many seconds of movies this mobile phone can take and send?) are the current target of differentiation. These differentiation and customization never ends because many people, young people in particular, tries hard to single them out.

These differentiation and customization leads to a complex, diverged network system.

\section{Fusion of Computer World and Communication World}

Link speed of networks is catching up that of computer's internal bus. Such excellent communication technologies are accelerating the fusion of computer world and communication world, which is another driving force that makes networks complicated and diverged.

Figure 1 shows the link speed of core telecommunication networks, networks that connect supercomputer nodes, and Ethernet. We can see that the link speed increase of networks is faster than that of supercomputers', and today the difference between supercomputer's internal bus and Ethernet is only one digit.

This is also true for common PCs. Gigabit Ethernet has the same speed with ordinary 32-bit PCI bus, and 10G-Ethernet is the same speed with PCI-X bus. Such high-speed WAN links are expensive, but a little bit modest one, 100Mbps FTTH is available at around $\$ 50 /$ month in Japan and more than $1.4 \mathrm{~m}$ customers are enjoying them.

This will make distributed computing into reality where communication functions and computer functions are tightly integrated. Technological reasons to distinguish RPC and ordinary system calls are diminishing. 


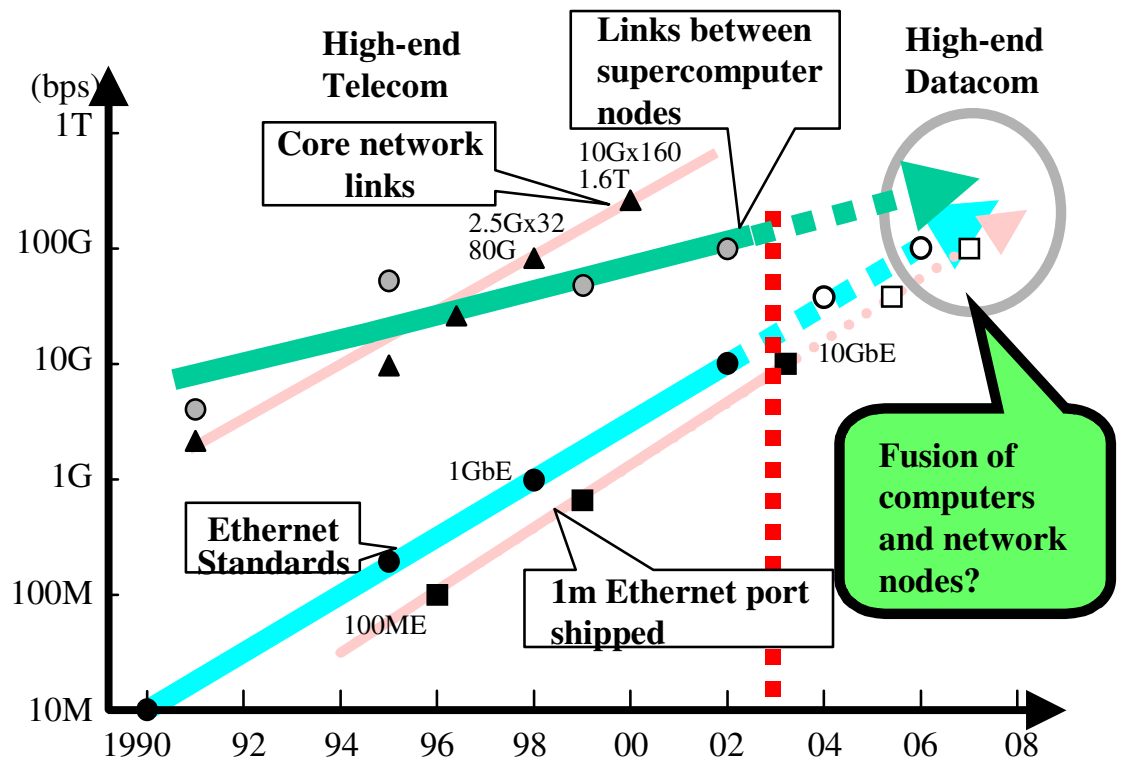

Fig. 1. Trends of link speed used in computers and telecommunications

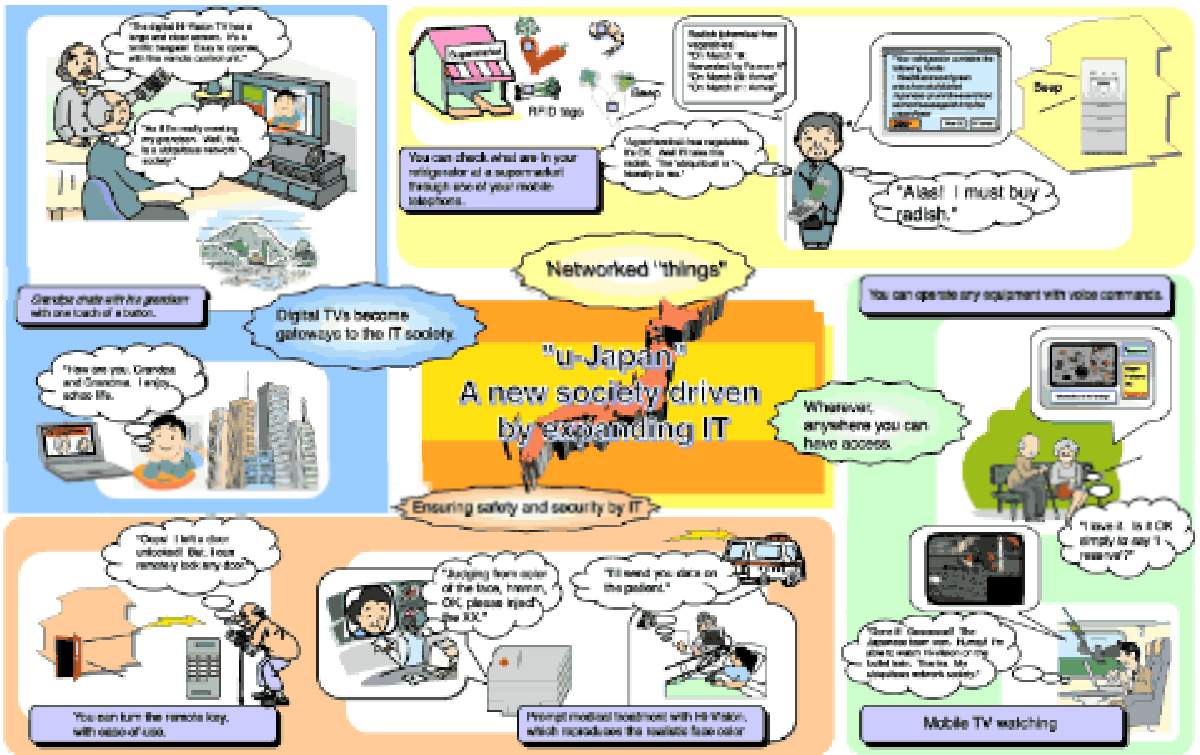

Fig. 2. U-Japan plan (cited from Japanese Government's white paper [4])

One example of distributed computing is various web services such as Amazon Web Services (AWS) [8]. AWS provides an API to search Amazon's bestsellers, prices, customer reviews and related items by using SOAP or XML. The search result 
can be used to decorate ordinary people's web sites, and enables them to sell Amazon's enormous kinds of goods on their web sites. Google provides a script that searches personal web sites as far as their contents are reachable from Google, and enables layman to use Google's mighty search power. These are the killer applications in the distributed computing era.

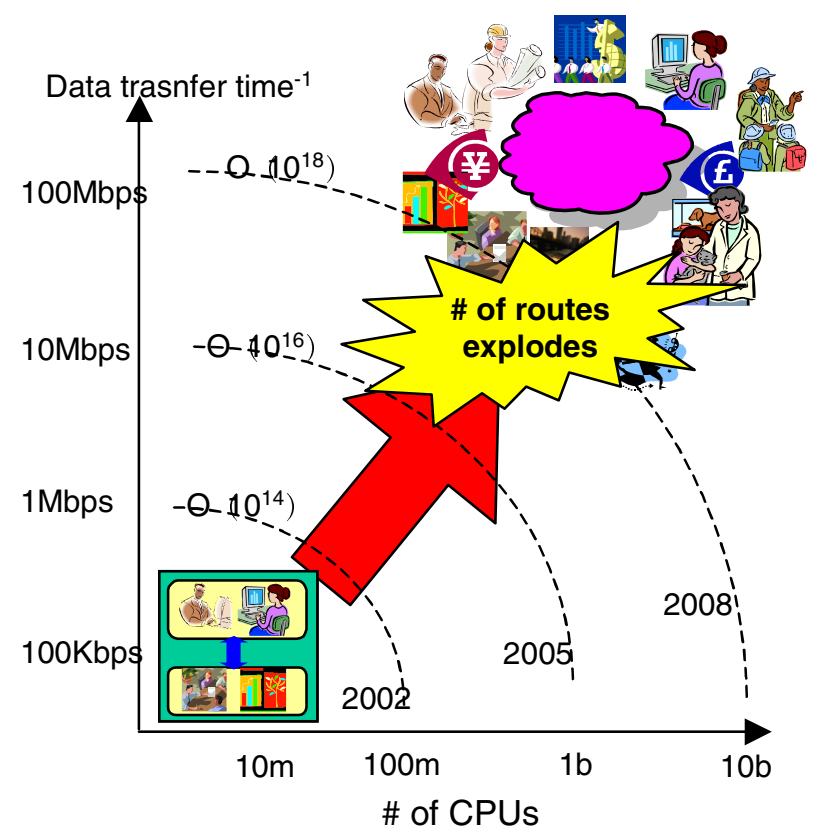

Fig. 3. Explosion of the number of status in future networks

Another example is ubiquitous computing. Japanese government is promoting 'u-Japan (Ubiquitous Japan)' [9] as the successor of e-Japan (a plan for an Internetready society). It describes a society where various servers, sensors such as RFIDs are connected to the network as shown in figure 2. We must solve various problems to build such networks. Security and privacy problems pointed out in [9] are of course important, but traditional problems such as routing also need to be solved. Figure 3 is a rough estimation of the number of CPUs connected to the Internet and the link speed between them, which shows that the number of connections between CPUs explodes by a factor of 100 or 10000 . Many nodes such as sensor nodes or PDAs will not have enough processing power to calculate optimal routes, and servers must calculate the routes on behalf of them. This means that it is difficult to apply clear endto-end model. Can we manage such a complicated large-scale network?

Current barrier that prevent such distributed computing is rather a social one, the right of ownership. Network operators cannot use customer's computing resources, vice versa. Management policy differs among different organizations. However, security threats are undermining this barrier. Cisco and IBM are cooperating to make security policies interoperable to protect systems from raging malwares, which means that $100 \%$ ownership is disappearing. 


\section{Tackling the Complexity}

Active network technologies have been trying to make network functions easier to customize and to arrange by enabling dynamic program installation, by making management system more sophisticated and by introducing security mechanisms against various threats. This matches the technological trends described above.

However, the authors fear that current active network technologies for solving complexity and dynamically changing nature of future networks might not be enough. We have developed various management systems, but were they enough if the number of nodes increased 10000 times because we had to consider sensor nodes and user terminals? Can we track the route of a sensor data to check its integrity? Can we detect the occurrence of security breaches in the network?

This is a challenging goal, and we believe theories build on solid mathematical basis, an approach that traditional active network research did not focus on, is indispensable for solving such issues of huge systems. Toward this end, mathematical methods and formulas developed in different areas will help us. Complex systems theory has a long history of research, and it provides various mathematical formulas such as self-organization and 'scale-free' [10][11]. As an example to show the potential power of such mathematical formula, we show that if we apply self-organization theories to future business environment, ubiquitous network is a must for system stability [13].

\subsection{The Theory of Scale-Free Networks}

Scale means a typical number that characterizes the system (e.g., mean). Scale-free systems such as fractal do not have such characteristic numbers (the mean becomes zero or infinity). The theory of scale-free claims that we can find scale-free networks in various areas, and it tries to explain the structure of World Wide Web, biochemical reaction chains of proteins in cells, and even the social network that propagates AIDS.

Let us take WWW as an example. In the World Wide Web network, there are few nodes such as Google or Yahoo that are linked from huge amount of nodes, while most nodes are linked from only a few links. Nodes such as Google and Yahoo are called 'hubs' or 'connectors', and play an important role in the networks. Figure 4 shows a typical image of such a network. You can see beautiful pictures of scale-free networks in various areas at [15].

To be more precise, it is known that the distribution of the number of links that a node has in the WWW network becomes power law distribution. If we denote the number of links node $v$ has as $d_{v}$, and the rank of node $v$ as $r_{v}$, we can describe the relationship between these two values as

$$
d_{v} \propto r_{v}^{\alpha}
$$

where $\alpha$ is a constant specific to the system.

This means that very few www nodes are linked from huge amount of nodes (i.e. hubs), but most nodes are linked from only a few nodes. 




Fig. 4. A typical scale-free network (inspired by a figure in [16])

Barabasi [10] showed analytically that such network appears if we assume two simple rules.

1. [Growth] a network grows by adding nodes one by one (the network does not appear at a time as a result of careful planning).

2. [Preferential attachment] the probability that a new node connect to an existing node is proportional to the number of links the existing node has (a famous node becomes even more famous).

Figure 5 shows the concept of such growth.

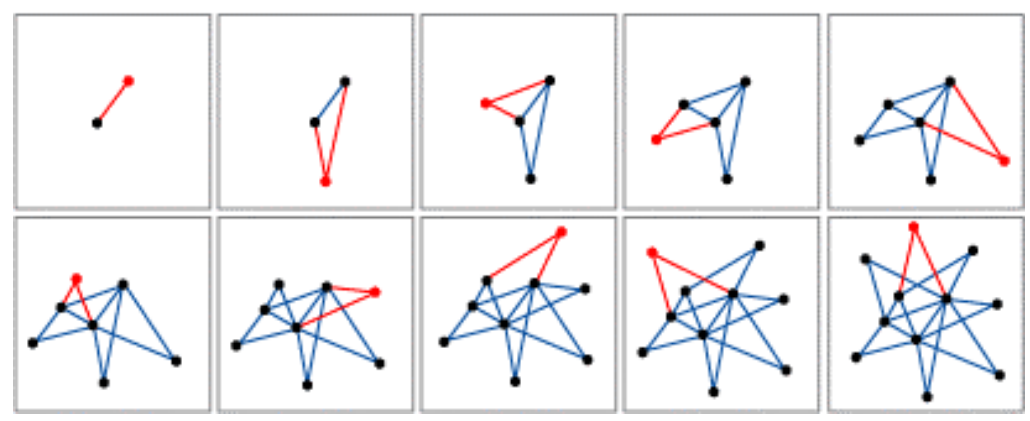

Fig. 5. Growth of a scale-free network (inspired by a figure in [11])

It has been proved that scale-free networks have many interesting properties. Scale-free networks are very robust against random failures, but fragile to intentional attacks [16]. They are also fragile to virus infection. According to percolation theory there is a minimum size of infected nodes to occur an epidemic in random networks, but in scale-free networks such threshold size does not exist [17]. Slight modification of the two rules, i.e. by adding the initial attractiveness of each web sites, changes the exponent $a$ of the Zipf's law [18]. This shows a possibility to control networks by the modification of 'attractiveness' of each nodes. 
There is an objection to this theory. Willinger [19] and Li [20] claim that preferential attachment is not the reason of IP networks' power-law distribution. They claim that the distribution is very common in complex systems because they are very 'robust' against various operations such as merging two groups, and it cannot be the proof of preferential attachment. Moreover, IP network is carefully designed considering various technological constraints, e.g., core routers cannot have many links. They conclude that Web network might be scale-free, but IP network is not. It is an open issue to find which claim (or still other claim) is true, and to build a formula for controlling networks on the findings.

\subsection{Self-organization in the Future Business Environment}

Now we would like to show that complex system theory such as self-organization can be used to describe the condition of complicated network stability, using networks for supply chain management (SCM) systems as an example.

SCM system builds a network among trade connections and by exchanging information on stocks it optimizes the stock of each company as a whole. Such business relations were stable and did not change very often in old days, but thanks to the Internet it becomes possible to establish relations with companies all over the world and change the trade connections for each trade.

Is this dynamically changing system stable?

Of course not, control theory tells us. The potential function of a system composed of many nodes becomes very complicated, and a little perturbation can shift the system to a new state, which often causes oscillation. We can see it as route flapping in the Internet.

However, there are many complex, still stable systems. Why?

Complex system theory claims that self-organization mechanisms reduce the number of dominant nodes in the system, and thanks to this degeneracy the potential function becomes simple, and the system becomes stable. If Yahoo and Google, or 'leader' or 'dominator' dominates the system, it becomes stable.

This phenomenon can be explained as follows [14]. System behavior can be described as a function of many parameters, each of which corresponds to each node. The time constant of each parameter differs. If some time constants are much larger than the others, the global behavior of the system can be described with these parameters. The parameters with large time constants are called 'order parameters'. This occurs because the behavior of nodes with small time constants changes before it affects the whole system, and its effect disperses. The behavior of these nodes can be described as a function of that of large time constants.

This means that if the time constants of the order parameters decrease, all other time constants must decrease to keep the system stable. If we would like to change a system more frequently every node must respond faster to various changes. Nodes require more sophisticated sensing system and communication tools to integrate the collected information.

And yes, this is the current trend in business world. To keep SCM systems every company are seriously working to respond more quickly to the changes of supply, stock, the environment, etc. To achieve this goal companies give their employee smart communication devices, and install various sensors in various places. The authors believe this is the reason why we need ubiquitous networks. 


\section{Conclusion}

We showed that the requirements that active network technologies tried to solve, the adaptation to network complexity and diversity, would become even more important in the future networking environment. They derive from the abundance of communication devices and the fusion of computer and communication technologies, and currently we do not see any sign that this trend would end in the near future. We then proposed to import knowledge from different areas, in particular complex systems theory to obtain mathematical basis to solve complicated network problem, and explained the necessity of ubiquitous networks.

There are huge amount of things to do for network engineers. The theory of scalefree is still in its infancy. It succeeded to describe and to explain various phenomena, but it does not say anything on what we can, and how we can control them. The explanation we made for ubiquitous networks is just a qualitative, introductory one, and more precise description is necessary to construct stable networks. We must go a long way to describe, explain and control various features of the Internet with this framework.

\section{References}

1. Jerome H. Saltzer, David P. Reed, and David D. Clark. End-to-end arguments in system design. ACM Transactions on Computer Systems 2, 4 (November 1984) pages 277-288. An earlier version appeared in the Second International Conference on Distributed Computing Systems (April, 1981) pages 509-512.

2. A. Barbir, E. Burger, R. Chen, S. McHenry, H. Orman, R. Penno, 'Open Pluggable Edge Services (OPES) Use Cases and Deployment Scenarios,' RFC3752, April 2004.

3. Border, J., Kojo, M., Griner, J., Montenegro, G. and Z. Shelby, "Performance Enhancing Proxies Intended to Mitigate Link-Related Degradations", RFC 3135, June 2001.

4. Walt W. Rostow, The Stages of Economic Growth: A non-communist manifesto, 1960.

5. 'Why phones are replacing cars,' the Economist, April 29, 2004.

6. M report, "Trends and forecasts of ring tone market", July 2003 (in Japanese).

7. Howard Rheingold, Smart Mobs: The Next Social Revolution, Perseus Books Group, October 2002.

8. http://www.amazon.com/gp/aws/sdk/main.html

9. Ministry of Public Management, Home Affairs, Posts and Telecommunications, Japan, 'Information and Communications in Japan', 2004 White Paper, 2004.

10. A.-L. Barabási, and R. Albert, 'Emergence of scaling in random networks,' Science,286, 509-512 (1999)

11. Albert-Laszlo Barabasi, Linked: How Everything Is Connected to Everything Else and What It Means, Plume Books; April 2003.

12. Koichiro Hayashi and Yoshihiro Tagawa, Universal service-fairness in multimedia era, Chuko-shinsho, March 1994 (in Japanese).

13. Takashi EGAWA, Masayoshi KOBAYASHI, Kenji YAMANISHI, Akira ARUTAKI and Junji NAMIKI, 'Dynamic Collaboration" from Scientists' Eyes,' NEC Journal of Advanced Technology, Vol.1, No.1 Winter 2004.

14. Hermann Haken, Synergetics: An Introduction, Springer-Verlag, June 1977.

15. http://www.nd.edu/ networks/gallery.htm 
16. R. Albert, H. Jeong, and A.-L. Barabási, 'Attack and error tolerance in complex networks,' Nature, 406, 387-482 (2000).

17. R. Pastor-Satorras and A. Vespignani, 'Epidemic dynamics and endemic states in complex networks,' Physical Review E 63, 066117, 2001.

18. S.N. Dorogovtsev, J.F.F. Mendes and A.N. Samukhin, 'Structure of Growing Networks: Exact Solution of the Barab'asi-Albert's Model,' Phys. Rev. Lett. 85, 4633, 2000.

19. W. Willinger, D. Alderson, J. Doyle, and L. Li, "More "Normal" Than Normal: Scaling Distributions and Complex Systems,' in Proceedings of the 2004 Winter Simulation Conference, 2004.

20. L. Li, D. Alderson, W. Willinger, and J. Doyle, 'A First-Principles Approach to Understanding the Internet's Router-Level Topology,' in Proceedings ACM SIGCOMM 2004, 2004. 